\title{
Soft Tissue Perivascular Epithelioid Cell Tumour: An Unusual Finding
}

Mauricio Bermúdez Sagre', Christian Ospina Pérez', Jennyfer Ortega Guatame ${ }^{2}$, Rosa Ospina Perez ${ }^{3}$, Ivan Lozada Martínez ${ }^{4}$, Jennifer Jiménez Valverde ${ }^{4}$, María Bolaño Romero ${ }^{4}$

${ }^{1}$ Surgery Department, University of Cartagena, Cartagena, Colombia;

${ }^{2}$ Surgery Department, Sinú University, Cartagena, Colombia;

${ }^{3}$ Medical Department, University Foundation of San Martin, Sabaneta, Colombia;

${ }^{4}$ Medical-Surgical Research Center, University of Cartagena, Cartagena, Colombia

Received April 26, 2020; Accepted April 30, 2021.

Key words: Perivascular epithelioid cell neoplasms - Sarcoma - Soft tissue neoplasms - Rare diseases - Case reports

Abstract: Perivascular epithelioid cell tumour (PEComa) is a rare mesenchymal tumour made up of clear perivascular cells with epithelioid characteristics, which co-expresses muscle and melanocytic markers with a component of spindle cells, like sarcoma and variety of other tissues. This time, we present the case of a young patient with a tumour in the dorsal region of progressive growth, compatible with PEComa of soft tissue after histopathological and immunohistochemical analysis.

Mailing Address: Ivan Lozada Martínez, MS., Medical-Surgical Research Center, University of Cartagena, Cartagena, Colombia; Phones: +57 31577998 23, +57 32155425 00; e-mail: ivandavidloma@gmail.com 


\section{Introduction}

Perivascular epithelioid cell tumour (PEComa) is a rare mesenchymal tumour made up of clear perivascular cells with epithelioid characteristics, which co-expresses muscle and melanocytic markers with a component of spindle cells, like sarcoma and variety of other tissues (Chen et al., 2016). It occurs more frequently in women in a 5:1 ratio compared to men, and with an average age of 45 years, benign behaviour prevails (Bao et al., 2019). These types of tumours have similar morphological and immunophenotypic characteristics, including epithelioid angiomyolipoma, lymphangioleiomyomatosis, and common clear cells (sugar tumour) (Lim et al., 2007; Bao et al., 2019), although malignant variants have also been described, mainly of gastrointestinal, gynecological, retroperitoneal, and uterine origin (Llamas-Velasco et al., 2016). Their presence in soft tissues is exceptional (Harris et al., 2004). Despite current advances in the biomedical sciences, no solid protocol has been established for the diagnosis or treatment of this specific disease. A hypothesis was recently proposed in which it is stated that perivascular epithelioid cells originate from pluripotent cells of the neural crest due to the expression of S-100, CD56, and CD-99 (Lim et al., 2007), which can give rise to cells smooth muscle and melanocytes, which may explain the histological pattern of this tumour. The objective of this article is to report the experience with a patient who presented a spontaneously appearing tumour in the dorsal region, who received standard definitive surgery, and subsequently found the unusual histopathological finding PEComa.

\section{Case report}

A 27-year-old female patient who consulted for a 3-month evolution disease manifesting with a tissue mass in the dorsal region of a progressive growth, without

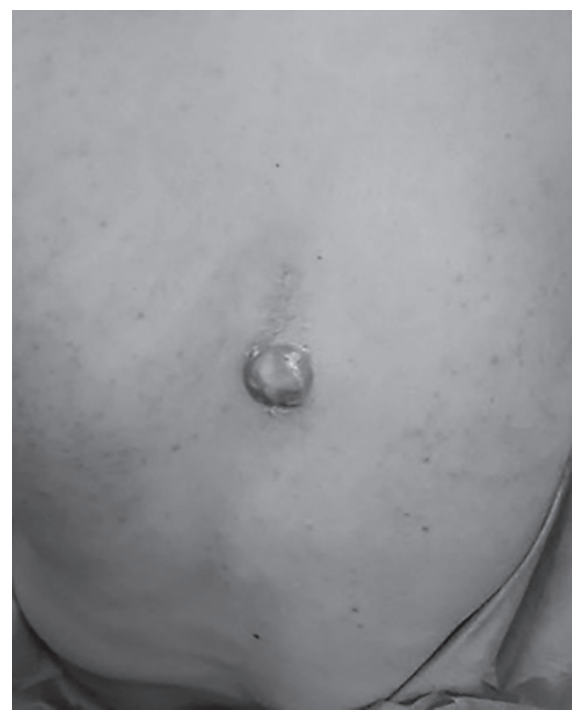

Figure 1 - Violaceous nodular lesion in the dorsal region that motivates consultation. 


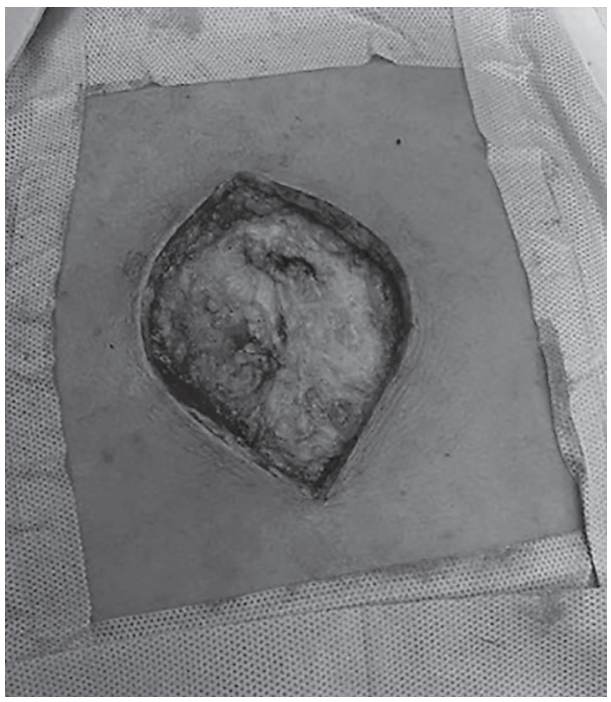

Figure 2 - Exeresis of tumour lesion.

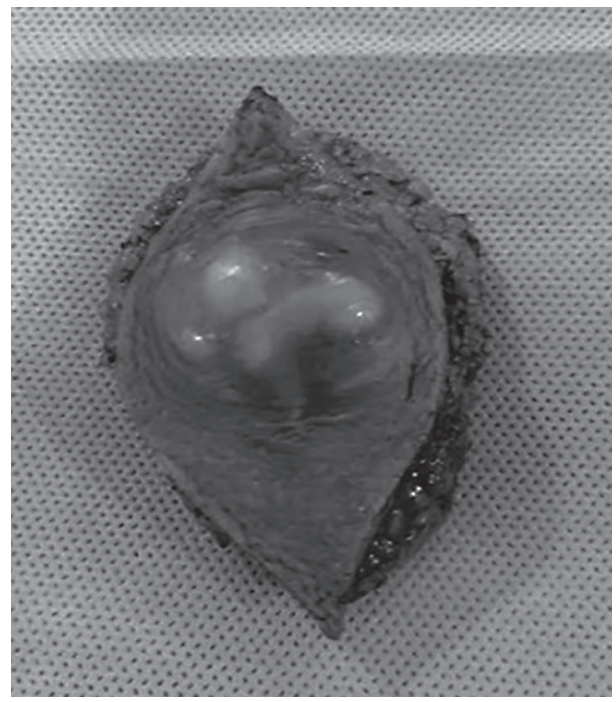

Figure 3 - Surgical piece resulting from the intervention.

pain, without bleeding, without weight loss. She reported that a year ago a similar lesion was resected in the same location, but the histopathological examination was inconclusive. There was not relevant pathological history. On physical examination, a raised nodular lesion at the level of skin was evident, it had firm consistency, $2 \mathrm{~cm} \times 3 \mathrm{~cm}$, was immobile, not painful, violet in colour at the location described (Figure 1). Based on the above, there was the diagnostic impression of a tumour with uncertain behaviour at the soft tissue level, so it was proposed to take the patient to surgery for excision of the lesion with flap.

This process was carried out without complications and extracted tissue was sent to pathology (Figures 2 and 3). During the postoperative period, she was a patient with good evolution, asymptomatic, with a healed surgical hound, without pain or bleeding. A pathology report described fibrohistiocytic lesion of uncertain behaviour with epithelioid areas and atypia, so immunohistochemistry procedures were performed, finding reactivity in Melan-A, MITF-1 and calponin, which suggested a definitive diagnosis of PEComa.

\section{Discussion}

PEComa was first described in the 1950s, wrongly reported as a renal angiomyolipoma (Morgan et al., 1951). In 2002, the World Health Organization recognized it for the first time as a heterogeneous mesenchymal tumour (Petersen, 2013), and subsequently, few isolated cases have been reported worldwide to date. It is considered the base of its origin in cell lines related to muscle and adipose 
tissue, having different patterns of intensity and sensitivity for the co-expression of melanocytic cells and muscle cell markers, this being one of its main characteristics (Folpe and Kwiatkowski, 2010).

These types of tumours are of extraordinary presentation, representing an incidence of less than 1\% of all benign tumours (Fenz et al., 2017), and their most frequent location are the adrenal glands (Kwazneski li et al., 2016), although they can be established from the retroperitoneum, bone, thyroid, lung, kidney, pancreas, liver, skin, uterus, gastrointestinal tract, etc. (D’Andrea et al., 2016; Ferrari et al., 2016; Fenz et al., 2017; Leal-Medrano et al., 2017; Touloumis et al., 2019; Zhao et al., 2019). These tumours have a nonspecific clinical presentation, being able to simulate any type of neoplasm, however, they usually debut with a painful sensation of mass, with typical characteristics of the affected organ (Cuevas et al., 2015), such as intestinal obstruction and bleeding in tumours of the gastrointestinal tract or bleeding vaginal in the case of uterine tumours.

Despite being considered mainly benign, those PEComas that are larger than $5 \mathrm{~cm}$, infiltrative growth pattern, high nuclear grade, cells with necrosis and atypical mitosis, are associated with malignancy (Folpe and Kwiatkowski, 2010; Schaefer and Fletcher, 2018). That is why the current diagnostic gold standard is through histopathology and immunohistochemistry, thus allowing to accurately examine the phenotypic characteristics of each particular case. For immunohistochemistry, CD-68 markers, S-100 protein, Calponin, cytokeratin AE1/AE3, epithelial membrane antigen (EMA), Human Melanoma Black-45 (HMB-45), Vimentin, anti-muscle smooth antibodies (SMA) and CD-117 are measured (Folpe et al., 2005; Petersen, 2013; Schaefer and Fletcher, 2018; Liu et al., 2019), which are taken by means of biopsy. Among the most common differential diagnoses are melanoma, sarcoma, epithelial tumours, and oxyphilic carcinomas (Petersen, 2013; Fenz et al., 2017), because melanosomes can be observed by ultrastructure, and a large amount of glycogen and cytoplasmic (Bao et al., 2019). The expression of this type of marker supports the hypothesis that PEComa is neoplasm originating from stem cells, which suffer defects acquired during differentiation.

So far, no specific treatment has been established, so it is resolved by surgical intervention, taking into account free margins of the tumour (Cuevas et al., 2015). It has a very good prognosis in the short and long term, since it is generally benign, but recurrence may occur if the resection margins of the lesion are not taken into account (Harris et al., 2004; Folpe et al., 2005).

In this case, fortunately the patient presented a benign neoplasm in a location that did not compromise any vital organ; the recommendations described as the resection of margins were taken into account when performing the operation, as well as the protocol to obtain the final diagnosis by means of immunohistochemistry, finding the expression of common markers registered in the literature. 


\section{Conclusion}

In this case, PEComa can be seen, a neoplasm of strange presentation, of uncertain behaviour. The tumor was studied with histopathological and immunohistochemical methods, to define the tumour phenotype and its association with malignancy. Reviewed literary data have shown, the need to take into account the resection margins that should be identified and avoided.

\section{References}

Bao, L., Shi, Y., Zhong, J., Zhao, M., Wu, J., Hai, L., Xu, X., Du, H., Shi, Y. (2019) Histopathologic characteristics and immunotypes of perivascular epithelioid cell tumors (PEComa). Int. J. Clin. Exp. Pathol. 12(12), 4380-4389.

Chen, Z., Han, S., Wu, J., Xiong, M., Huang, Y., Chen, J., Yuan, Y., Peng, J., Song, W. (2016) A systematic review: Perivascular epithelioid cell tumor of gastrointestinal tract. Medicine (Baltimore) 95(28), 1-7.

Cuevas, O., Escobar, L., Rodriguez, M., Artigas, V. (2015) PEComa, a rare epithelioid cell tumor. Cir. Esp. 93(7), e65-e67.

D’Andrea, D., Hanspeter, E., D’Elia, C., Martini, T., Pycha, A. (2016) Malignant perivascular epithelioid cell neoplasm (PEComa) of the pelvis: a case report. Urol. Case Rep. 6, 36-38.

Fenz, L., Mehrmann, M., Kremp, K., Schneider, G. (2017) Soft tissue tumors: Epidemiology, classification and staging. Radiologe 57(11), 973-986. (in German)

Ferrari, A., Dirksen, U., Bielack, S. (2016) Sarcomas of soft tissue and bone. Prog. Tumor Res. 43, 128-141.

Folpe, A., Kwiatkowski, D. (2010) Perivascular epithelioid cell neoplasms: Pathology and pathogenesis. Hum. Pathol. 41(1), 1-15.

Folpe, A., Mentzel, T., Lehr, H., Fisher, C., Balzer, B., Weiss, S. (2005) Perivascular epithelioid cell neoplasms of soft tissue and gynecologic origin: A clinicopathologic study of 26 cases and review of the literature. Am. J. Surg. Pathol. 29(12), 1558-1575.

Harris, G., McCulloch, T., Perks, G., Fisher, C. (2004) Malignant perivascular epithelioid cell tumour (“PEComa”) of soft tissue: a unique case. Am. J. Surg. Pathol. 28(12), 1655-1658.

Kwazneski li, D., Merrill, M., Young, J., Sell, H. Jr. (2016) Angiomyolipoma and malignant PEComa: Discussion of two rare adrenal tumors. Case Rep. Oncol. Med. 2016, 5204092.

Leal-Medrano, J. A., Marín-Hernández, L. R., Castellanos Bueno, R., García Ayala, E. (2017) PEComa (neoplasia de células epiteliodes perivasculares) asociado con cáncer papilar de tiroides bilateral sincrónico. Rev. Chil. Cir. 69(6), 483-488.

Lim, S. D., Stallcup, W., Lefkove, B., Govindarajan, B., Au, K. S., Northrup, H., Lang, D., Fisher, D. E., Patel, A., Amin, M. B., Arbiser, J. L. (2007) Expression of the neural stem cell markers NG2 and L1 in human angiomyolipoma: Are angiomyolipomas neoplasms of stem cells? Mol. Med. 13(3-4), 160-165.

Liu, C. H., Chao, W. T., Lin, S. C., Lau, H. Y., Wu, H. H., Wang, P. H. (2019) Malignant perivascular epithelioid cell tumor in the female genital tract: Preferred reporting items for systematic reviews and meta-analyses. Medicine (Baltimore) 98(2), 1-7.

Llamas-Velasco, M., Requena, L., Mentzel, T. (2016) Cutaneous perivascular epithelioid cell tumors: A review on an infrequent neoplasm. World J. Methodol. 6(1), 87-92.

Morgan, G. S., Straumfjord, J. V., Hall, E. J. (1951) Angiomyolipoma of the kidney. J. Urol. 65(4), 525-527.

Petersen, I. (2013) The new WHO classification and recent results in soft tissue tumor pathology. Pathologe 34(5), 436-448. (in German)

Schaefer, I. M., Fletcher, C. (2018) Recent advances in the diagnosis of soft tissue tumours. Pathology 50(1), $37-48$. 
Touloumis, Z., Giannakou, N., Sioros, C., Trigka, A., Cheilakea, M., Dimitriou, N., Griniatsos, J. (2019) Retroperitoneal perivascular epithelioid cell tumours: A case report and review of literature. World J. Clin. Cases 7(21), 3524-3534.

Zhao, J., Teng, H., Zhao, R., Ding, W., Yu, K., Zhu, L., Zhang, J., Han, Y. (2019) Malignant perivascular epithelioid cell tumor of the lung synchronous with a primary adenocarcinoma: One case report and review of the literature. BMC Cancer 19(1), 1-5. 